\title{
A SMART MEDIKIT USING IOT TECHNOLOGY
}

\author{
Alen John Thomas ${ }^{1}$, Abhishek Radhakrishnan ${ }^{2}$, Arun Geroge ${ }^{3}$, Geo Joy ${ }^{4}$, Gayathri R Krishna ${ }^{5}$ \\ ${ }^{1}$ Mangalam College of Engineering, India, alen4akhil@gmail.com \\ ${ }^{2}$ Mangalam College of Engineering, India, abhishekrak97@gmail.com \\ ${ }^{3}$ Mangalam College of Engineering, India, arungeorge6297@ gmail.com \\ ${ }^{4}$ Mangalam College of Engineering, India, itsmegeo1997@gmail.com \\ ${ }^{5}$ Mangalam College of Engineering, India, gayathri.krishna@mangalam.in
}

\section{ABSTRACT}

In today's busy world people may not always be in a position to keep them self updated of their medicine timings. The course of the medicine is often forgotten by patients, which affect their sickness and may even lead to conditions such as paralyzation, coma, or even death. A new device named SMART MEDIKIT will help in solving this current issue. This device is an efficient system that replaces the current problem of paralyzed people who are on daily medication. The proposed system helps the aged people to take their medicines on time without the help of another person. It is a box type small system equipped with Internet of Things (IoT) and consists of seven chambers one for each day in a week. Medicines are kept in these chambers and there is a facility to set the time in which the person has to take the medicine. According to the time that is set on the device there will be a buzzer to alert about the medicine. After the buzzer, the chamber will automatically open and the patient can take their medicine. Once the chamber closes an intimation message will be sent to the close relative and family doctor via any social medium. Also an emergency button is present on top of the box to inform the doctor and close relatives about patient and if any.

Key words: Internet of Things (IoT), Radio Frequency Identification (RFID), Internet of Medical Things (IoMT), Controlled Delamination Material(CDM).

\section{INTRODUCTION}

Today healthcare is a big problem faced by the population, due to existence of chronic diseases, and the high cost in medicine etc. Aging populations and the chronic diseases takes up a large amount of healthcare resources. A number of device manufacturers and other players are trying to tackle the problem of handling the medication of old people. This can be done by using the concepts of IoT, Android Application and cloud computing in patient's or his caretaker's smart phone, by which they can view the current health status of the patient.

\section{LITERATURE SURVEY}

The SMART MEDIKIT is a box that can help people to take medicines on time. This kit will remind the patient about the medicines according to the time set on the system and partially paralyzed patients can take medicines without the help of another person. This system is more beneficial to patients who are suffering from the disease such as Alzheimer. It is provided with an LCD display to notify the dosage and name of the medicines and also with an emergency switch that will help the patient in any emergency situation.[1]

Figure 1 describes about the block diagram of Medikit. It consist of an LCD display which notifies the dosage and name of the medicines and it is also provided with an emergency switch that will help the patient in any emergency situation. The emergency switch is associated with a GSM module through which nearest hospitals and ambulance can be informed that a person needs help in a particular region. The system consist of an AVR microcontroller which is the main part of the system. The system is secured with password so that any unauthorized person cannot make any changes in the system. It also contains a real time clock to evaluate a bell time that we set on the system with the real time. When the bell time and the real time matches buzzer 
will announce to take medicine and the buzzer sound will remain as long as the medicine is taken from the automatically opened chamber.

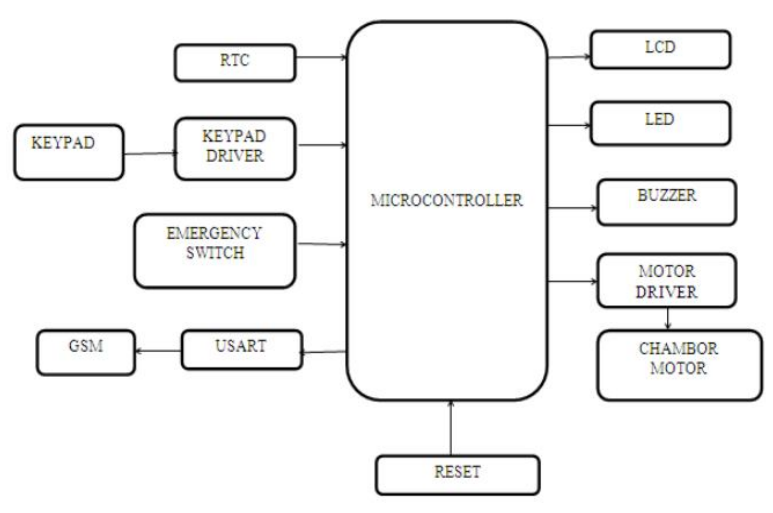

Figure 1: Block diagram of MEDIKIT

The number of medical equipment' and their complexity force hospitals to adopt different maintenance strategies to enhance the performance of their devices in addition to attempting to reduce their maintenance cost and effort. A predictive maintenance strategy that relies on real online data through using the Internet of Things (IoT) technology to predict failure before it occurs and the remaining useful life of medical equipment as well.

In terms of architecture, it consist of three layers as illustrated in Figure 2. which are: Perception layer, Network layer, and Application layer[2].

The technologies of home healthcare, which are currently used for improving the situation, by reminding the schedule of medicine, remote monitoring and updating new datas of patients, which can be done by doctor through web. Internet of Things (IoT) network will provide active and realtime appointment of patient, hospitals, caretaker and doctors. Apart from this a secured data transmission is required for the purpose of remote monitoring, and hence there is need of the architecture of a low cost embedded platform for Web-based monitoring [3].

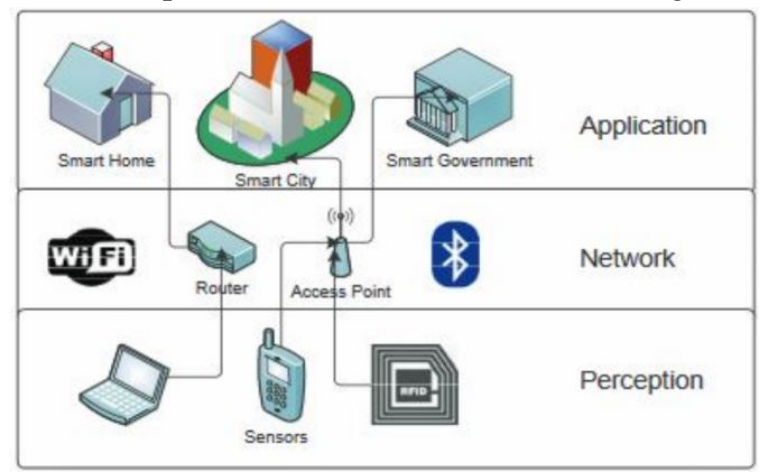

Figure 2: Three-layer IoT architecture

A pervasive and preventive healthcare solution for medication noncompliance and daily monitoring is proposed using an intelligent package sealed by Controlled Delamination Material (CDM) and controlled by Radio Frequency Identification (RFID. Pervasive healthcare has been widely approved to be the next generation form of healthcare, in which distributed, patient-centric and self-managed care is emphasized comparing to the traditional hospitalized, staff-centric and professional managed care. Firstly, preventive strategies are provided using an intelligent package sealed by Controlled Delamination Material (CDM) and controlled by Radiofrequency Identification (RFID)[4].

Internet of Things (IoT) technology has gained much attention in recent years for its potential to alleviate the strain on healthcare systems caused by an aging population and a rise in chronic illness. Standardization is a key issue limiting progress in this area, and thus this paper proposes a standard model for application in future Internet of Things healthcare systems. It also presents the state-of-theart research relating to each area of the model, evaluating their strengths, weaknesses, and overall suitability for a wearable IoT healthcare system[5].

This paper presents a design and implementation of an IoT-based health monitoring system for emergency medical services which can demonstrate collection, integration, and interoperation of IoT data flexibly which can provide support to emergency medical services like Intensive Care Units (ICU), using a INTEL GALILEO 2ND generation development board. The proposed model enables users to improve health related risks and 
reduce healthcare costs by collecting, recording, analyzing and sharing large data streams in real time and efficiently [6].

Existing processes for patients' vital data collection require a great deal of labor work to collect, input and analyze the information. A solution is made to automate this process by using "sensors" attached to existing medical equipments that are inter-connected to exchange service. The proposal is based on the concepts of utility computing and wireless sensor networks [71.

\section{PROPOSED SYSTEM}

\section{Components of the proposed system are:}

\section{1) Raspberry Pi}

Raspberry Pi is a credit card sized small computer which can be capable of doing almost all functions of a personal computer including internet surfing, playing games, watching movies etc.

\section{2) Lock system for Medikit}

Medikit consists of 7 compartments for different days in a week. Lock system can be operated by raspberry pi to automatically open the particular chamber for a day.

\section{3) Chamber close alert}

Chamber should close manually. After a definite time limit if the box is not closed, then an alert will sent to the patient and the relatives.

\section{4) Buzzer/ speaker unit}

While any of the compartments opened automatically, buzzer unit alerts the patient. This beep sound will stop only after closing that compartment. The speaker associated with this unit will announce the medicine details for that particular time.

\section{5) Emergency switch}

Emergency switch associated with this system will help the patient to inform relatives and family doctor in emergency situations. While pressing this button predefined messages will be send to relatives and doctor via telegram.

\section{6) Magnetic Reed switch}

The proposed Magnetic Reed switches are rugged in operation which is operated by means of stepper motors. The stepper motors are having controlled signal from Raspberry pi. Earlier nobody has used the sensor in patient monitoring systems. The schematic view of magnetic reed switches is Figure 3

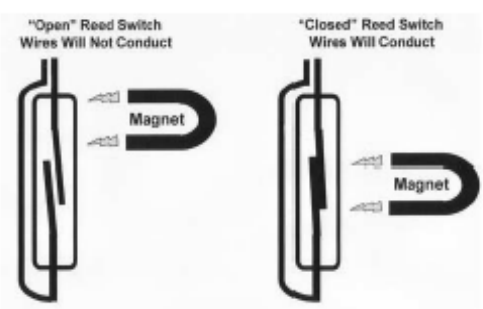

Figure 3: Magnetic Reed switches

In the proposed work, the three compartments are opened/closed by these switches by means of electrical signal from the pi controller.The block diagram of the proposed system is shown in figure 4 .

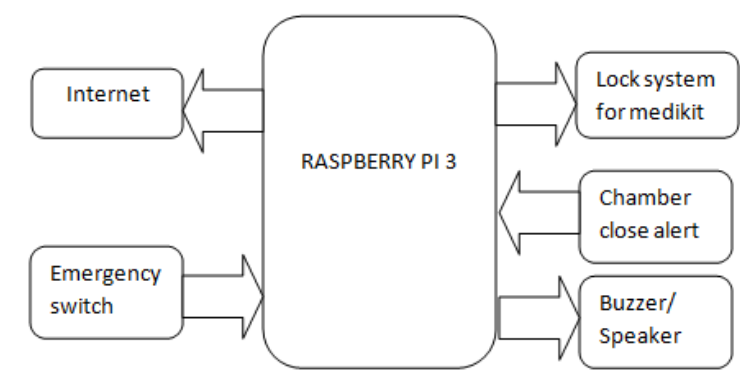

Figure 4: Block diagram of the proposed system

\section{MODULE DESCRIPTION}

\section{ADMIN}

This module is used to set the medicine types and time according to the doctor's prescription. Main roles of this are adding medicine details, time, dosage etc. It also sets a predefined message.

\section{DOCTOR}

Doctor will treat the patients and provides the medicine. The box is filled with medicine that is prescribed by the doctor.

\section{PATIENT}

The patient should take medicine when the box opens. They receive an alert message to take the medicine. 
Alen John Thomas et al., International Journal of Networks and Systems, 8(3), April - May 2019, 75 - 78

\section{RELATIVE}

Relative can view the medicine details that are prescribed by the doctor. They fill medicines in the box according to the doctor's prescription. If the patient doesn't take the medicine in prescribed time, the relatives will receive a message through telegram app.

\section{IMPLEMENTATION}

A modern healthcare IoT platform with an intelligent medicine box along with sensors for health monitoring and diagnosis is implemented. An intelligent home-based medicine box with wireless connectivity along with an android application helps patients and doctors to be in close communication. The proposed platform has an intelligent medicine box that gives alerts to patients to take their medicine at the right time. The box is connected to the internet to make timely updates about the consumption of medicines by the patient to the already registered mobile number. The entire setup is shown in Figure 5

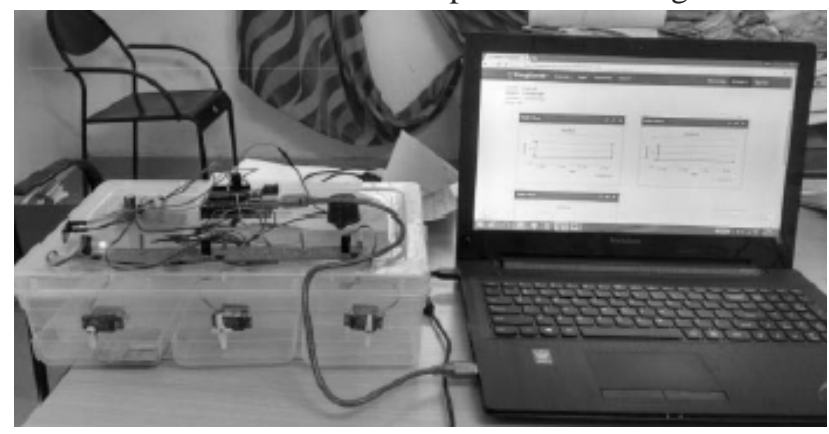

Figure 5: Working model of Smart MEDIKIT

\section{CONCLUSION}

Smart Medikit using IoT technology helps the hospital authorities to continuously monitor the patients. It also helps the patient to remind about the medicines in time. So the doctor can have direct view over his patient. Thus the medication procedures can be shifted from hospital to home. Earlier the medicine box was proposed with Ultrasonic sensors, IR sensors and Weight based sensors. But these sensors have less reliability because of ambient light and improper calibration. So, the proposed system uses Magnetic Reed switches which are operated by means of stepper motors. The stepper motors are having controlled signal from Raspberry pi.

\section{REFERENCES}

[1].Anju Raphel, Binimol Benny, Dincy Mathew, DrisyaSukumaran Nair, Anita George ,An efficient system for medication: Smartmedikit, International Journal of Advanced Research in Electrical, Electronics and Instrumentation Engineering,Vol. 6, Issue 3, March 2017

[2].Jumana Farhat, Abdulrahim Shamayleh, and Hasan Al-Nashash ,Medication equipment efficient failure management in iotenvironment,American University of Sharjah, Sharjah, United Arab Emirates,may 2017.

[3].SamirV.Zanjala, Girish. R. Talmaleb,M.E. Medicine reminder and monitoring system for secure health using iot,International Conference on Information Security \& Privacy (ICISP2015), 11-12 December 2015.

[4].Zhibo Pang, Qiang Chen, LirongZhengiPackVinn A pervasive and preventive healthcare solution for medication noncompliance and daily monitoring, iPack Vinn Excellence Center, School of Information and Communication Technology Royal Institute of Technology (KTH).

[5].Stephanie Baker, Wei Xiang, Senior Member, IEEE, and Ian Atkinson.Internet of things for smart health care: technologies, challenges, and applications

[6].Punit Gupta1, Deepika Agrawal2, Jasmeet Chhabra3, Pulkit Kumar ,IoT based smart healthcare kit, International Conference on Computational Techniques in Information and Communication Technologies (ICCTICT),2016. https://doi.org/10.1109/ICCTICT.2016.7514585

[7].Carlos Oberdan Rolim, Fernando Luiz Koch, Carlos Becker Westphall, Jorge Werner, Armando Fracalossi, Giovanni Schmitt Salvador, Acloud computing solution for patient's data collection in health care institutions, Network and Management Laboratory - LRG Federal University of Santa Catarina Florianopolis - SC - Brazil. 